\title{
Non-elective and Revision Arthroplasty Are Independently Associated With Hip and Knee Prosthetic Joint Infection Caused by Acinetobacter Baumannii: a Brazilian Single Center Observational Cohort Study of 98 Patients
}

\section{Raquel Bandeira Silva}

Hospital São Francisco de Assis

Rodrigo Otavio Araujo

Hospital São Francisco de Assis

Mauro José Salles ( $\nabla$ salles.infecto@gmail.com )

Santa Casa de São Paulo School of Medical Sciences

\section{Research Article}

Keywords: Acinetobacter baumannii, multidrug-resistant, arthroplasty infection, risk factors, DAIR, treatment failure

Posted Date: April 22nd, 2021

DOI: https://doi.org/10.21203/rs.3.rs-437337/v1

License: (9) (i) This work is licensed under a Creative Commons Attribution 4.0 International License. Read Full License 


\section{Abstract}

Background: Prosthetic joint infection (PJI) caused by Acinetobacter baumannii $(A b)$ has become a growing concern due to its overwhelming ability to express resistance to antibiotics and produce biofilm.

Aim: This study aimed to identify independent risk factors (RFs) associated with $A b$-PJI and its role in the treatment outcome.

Methods: Single-center retrospective, cohort study of PJI patients diagnosed between January 2014 and July 2018. PJI diagnosis was based upon MSIS 2018 criterium. To estimate RFs associated with $A b$-PJI, multivariate analyses with level of significance of $p<0.05$ was performed. To evaluate treatment failure, the Kaplan-Meier (KM) analysis and log-rank test was performed.

Results: Overall, 98 PJI cases were assessed, including 33 with $A b$-PJI and 65 with PJI due to other microorganisms (Non- $A b$-PJI). Independent RFs associated with $A b$-PJI were revision arthroplasty (odds ratio $[\mathrm{OR}]=3.01 ; 95 \%$ confidence interval $[95 \% \mathrm{Cl}]=1.15-7.90, \mathrm{p}=0.025)$ and non-elective arthroplasty $(\mathrm{OR}=2.65 ; 95 \% \mathrm{Cl}=1.01-7.01, \mathrm{p}=0.049)$. $A b$-PJI was also more likely to be classified as a chronic late infection $(\mathrm{OR}=5.81 ; 95 \% \mathrm{Cl}=2.1-16.07, \mathrm{p}=0.001)$ than Non- $A b$-PJI. $A b$-PJI was not associated with treatment failure $(p=0.557)$.

Conclusions: Late chronic infections, surgical revision, and non-elective arthroplasty are well-known predictors of PJI, but were also independently associated with $A b$-PJI. High selective pressure imposed by misuse of antibiotics is likely to have played a role. Infections caused by $A b$ and surgical treatment with DAIR were not associated with PJI treatment failure.

Trial registration: Study data supporting our results were registered at an open access virtual platform for registration of studies on humans performed in Brazil. The Brazilian Registry of Clinical Trials (ReBEC) http://www.ensaiosclinicos.gov.br/rg/RBR-6ft5yb/

\section{Register Number: RBR-6ft5yb}

\section{Introduction}

Worldwide, an increasing number of individuals have undergone joint replacement surgeries particularly at the hip and knee, either for elective reasons or following sustained trauma. Among the possible complications, prosthetic joint infection (PJI) is the most feared despite the low incidence ranging between $1-2 \%[1,2]$ for primary and up to $4 \%$ for revision surgeries $[3,4]$, but with high morbidity and mortality rates. Gram-positive cocci (GPC), such as Staphylococcus aureus and coagulase-negative Staphylococci are the major PJI-related microorganisms followed by gram-negative bacilli (GNB), with prevalence ranging from 5-23\% [4-6]. In some case series, PJI due to GNB have been reported at rates greater than 40\%. [7, 8]

Acinetobacter is a genus of gram-negative bacteria comprising 31 different species. Due to its ability to spread in healthcare environments, Acinetobacter baumannii $(A b)$ is currently the most difficult species to 
control and eradicate.[9] This microorganism is ubiquitous in the environment [9] and has become one of the most successful pathogens associated with healthcare-related infections due to its ability to express a variety of antimicrobial resistance mechanisms and to form biofilms on both biotic and abiotic surfaces. [10] On a global scale, approximately $50 \%$ of $A b$ strains have been identified as multidrug-resistant (MDR). The World Health Organization (WHO) has declared carbapenem-resistant $A b$ to be one of the most important species among the group of bacteria termed ESKAPE (Enterococcus faecium, Staphylococcus aureus, Klebsiella pneumoniae, A. baumannii, Pseudomonas aeruginosa, and Enterobacter species), which are considered priority pathogens due to the threat they pose to global public health, requiring urgent actions and the development of new antibiotics to combat them.[11, 12] Unfortunately, among several Latin American countries, $A b$ strains have shown resistance to virtually all classes of antibiotics, including carbapenems. This worrisome microbial epidemiology has been identified at some Brazilian hospitals, where $77 \%$ of these isolates have exhibited resistance to carbapenems.[13] The production of oxacillinhydrolysing carbapenemase (carbapenem-hydrolysing class D enzymes) has been identified as the most common antibiotic resistance mechanism, and the global dissemination of OXA-type clones, including OXA-23, OXA-72, and OXA-58, is regarded as the most common mechanism of antibiotic resistance $[13,14]$

The emergence of musculoskeletal surgical site infections (SSIs) and orthopaedic implant-associated infections caused by $A b$ has become a matter of urgent concern for healthcare providers due to the limited therapeutic arsenal available, particularly against carbapenem-resistant strains.[15] Moreover, treatment of PJI caused by MDR and extensively drug-resistant (XDR) GNB, particularly $A b$, is hampered by its ability to be encased within biofilms. The resistance of $A b$ against virtually all antimicrobials and its intrinsic capacity for biofilm formation may be associated to lower cure rates and increase disease morbidity, since treatment usually requires a combination of highly toxic systemic antibiotics $[16,17]$ Despite this challenge, to our knowledge, no published studies investigated independent risk factors (RFs) for the $A b$ PJI. Indeed, few previous publications on $A b$-PJI attempted to describe in case-series report format, aspects of surgical and antibiotic therapy $[15,18,19]$. Therefore, this study aimed to identify the independent RFs for $A b P J I$ and assess the role of $A b$ on the treatment outcome.

\section{Materials And Methods}

\section{Study design}

This study was performed as an observational, single-centre, retrospective, cohort study using data obtained from 2,672 patients undergoing arthroplasties between January 2014 and July 2018, at a Brazilian orthopaedic referral center. All patients diagnosed with PJI, either due to $A b(A b-\mathrm{PJI})$ and other microorganisms (Non- $A b$-PJI), were identified from clinical and microbiological records and surgical description sheets. The primary study endpoint was the identification of independent predisposing factors associated with PJI caused by $A b$ and secondary endpoint was to access if the AbPJI have influence on treatment outcome. The study included individuals aged 18 years or older who met the diagnosis criteria for PJI according to the Musculoskeletal Infection Society (MSIS) [20]. Inclusion criteria also required the same identified pathogen yielding in at least two peri-prosthetic tissue samples, and prospective follow-up 
period of a minimum one-year period. Patients who underwent arthroplasty at an institution other than ours, did not meet the criteria for PJI as defined by the MSIS or had culture-negative results were excluded. The study was reviewed and approved by the local ethics committee (approval no. 2,610,914 on April 20, 2018).

\section{Definitions}

The PJI onset date was defined according to the date of the first observation of typical infectious signs and symptoms. MDR- $A b$ was defined as the nonsusceptibility of the identified pathogen to at least one antimicrobial agent from three or more different antimicrobial classes (e.g., aminoglycosides, cephalosporins with an anti-Pseudomonas effect, carbapenems, fluoroquinolones, penicillin $+\beta$-lactamase inhibitors, monobactams and polymyxin). $A b$ that were extensively drug-resistant (XDR) to multiple antibiotics were defined as those lacking susceptibility to at least one antimicrobial agent from all but two classes of antimicrobials [21]

Early-onset PJI was defined as those cases occurring $<3$ months after the index surgery, whereas late PJI was defined as those cases in which the diagnosis occurred more than 3 months after the index surgery. The remission of infection was defined as the absence of clinical, laboratory, or radiological symptoms at the last medical follow-up (with a minimum follow-up time of one year). Therapeutic failure was defined as infection recurrence at a previously controlled site; requirement for new surgery, a second course of antimicrobial therapy, chronic antibiotic suppression, excision arthroplasty, or limb amputation; or death within the follow-up period $[22,23]$

\section{Microbiological analysis}

In the surgical ward, a minimal of three different periprosthetic tissue samples and synovial fluid were collected and processed for microbiology. Synovial fluid sample were aseptically inoculated into aerobic standard blood culture bottles. Tissue samples were homogenised in $3 \mathrm{ml}$ of brain-heart infusion (BHI) broth for $1 \mathrm{~min}$ and inoculated onto aerobic sheep blood agar, chocolate agar, and anaerobic blood agar and into thioglycolate broth (BD Diagnostic Systems, Sparks, MD). The time limit for processing samples was 6 hours. Aerobic were incubated aerobically at $35-37^{\circ} \mathrm{C}$ in $5-7 \% \mathrm{CO}_{2}$ for 7 days, and anaerobic plates were incubated at $37^{\circ} \mathrm{C}$ for 14 days. Additionally, $0.5 \mathrm{ml}$ of tissue homogenate was inoculated in thioglycolate broth, incubated for 14 days, and sub-cultured on blood agar plates when the broth became cloudy. Colonies of microorganisms growing on plates were identified, and their susceptibilities to antibiotics were tested according to standard microbiological techniques. The bacteria were identified by conventional biochemical and metabolic tests in accordance with the international standards and definitions established by the European Committee on Antimicrobial Susceptibility Testing (EUCAST) [24]. Sensitivity tests were performed using the disk diffusion technique, and the determination of minimum inhibitory concentrations (MICs) was performed by automated means or by the e-test method, the results of which are presented according to standardised microbiological techniques.

\section{Potential risk factors}


Variables associated with the patient, surgery, and postoperative procedures were identified by reviewing the medical, intraoperative, and microbiological records to identify potential RFs for $A b$-PJI. Demographic variables (sex and age), comorbidities (the presence and number of comorbidities, alcoholism, and smoking habits), the American Society of Anaesthesiologists (ASA) physical status classification, previous use of antibiotics during the past three months, and previous orthopaedic infections were assessed. Associated surgical aspects included the arthroplasty site (hip vs knee), total or partial arthroplasty, primary or revision surgery, and post-traumatic arthroplasty or elective arthroplasty. The factors related to the postoperative period that were considered included postoperative hematoma, the presence of sepsis at the time of diagnosis, concomitant infections diagnosed at different sites, and early or late infection. The surgical strategies, including debridement, antibiotics, and implant retention (DAIR) or any prosthesis removal (Non-DAIR), were assessed for survival and outcome analyses.

\section{Statistical analysis}

For the overall study population and the groups defined as $A b$-PJI and Non- $A b$-PJI, qualitative variables are reported as the mean and percentage, and quantitative variables are presented as the median and standard deviation (SD). Associations between qualitative variables were analysed using the Chi-square test and Fisher's exact test, as indicated. The associations between quantitative variables were assessed by logistic regression. The risk estimate was calculated for the associated variables and reported as the odds ratio (OR) with a 95\% confidence interval $(\mathrm{Cl})$. The logistic regression model was used to select significant variables from among those identified as significant in univariate analyses. Only variables with significance less than $0.20(p<0.20)$ were included in the logistic regression. Variables with significance less than $0.05(p<0.05)$ in the multiple regression were included in the final model. To estimate the probability of survival as a function of time, Kaplan-Meier (KM) analyses were performed, and the resulting curves were compared using the log-rank method. All data were analysed using SPSS, version 23 (IBMSPSS Inc., Chicago, IL, USA).

\section{Results}

A total of $115 \mathrm{PJI}$ cases were assessed for inclusion in the study, of which, 14 cases that did not meet the MSIS criteria for infection and 3 cases with less than two or negative tissue cultures were excluded. Therefore, 98 PJI cases were analysed, 33 in the $A b$-PJI group and 65 in the Non- $A b$-PJI group.

\section{Study population:}

The demographic and clinical characteristics of the study population are summarized in Additional file 1. Most PJI patients were females (58.16\%), with a mean age of 67.3 years (SD \pm 13.2$)$. Interestingly, hip arthroplasty was the most frequent procedure (83.7\%). Over $70 \%$ of patients had at least one comorbidity, among which hypertension (61.2\%) and diabetes mellitus (20.4\%) were the most common. Arthroplasty was primary surgery in $57.1 \%$ (56/98) of cases and due to fractures (non-elective) in $39.8 \%$ (39/98). PJI was classified as early in $69.4 \%(68 / 98)$ and $19.4 \%$ (19/98) had a previous PJI (Additional file 1)

\section{Microbiology}


Among 33 patients with $A b$-PJI, 27 strains were classified as $A b$-XDR, 4 as $A b$-MDR, and only $2 A b$ strains were sensitive to multiple antibiotics ( $A b-\mathrm{MS})$. Although the susceptibility to carbapenem was below $6 \%$, all isolates were $100 \%$ susceptible to colistin (Table 1).

Table 1

Antibiotic susceptibility of Acinetobacter baumannii strains

\begin{tabular}{|c|c|c|c|c|c|c|c|c|c|c|c|}
\hline Antibiotics & SPT & CIP & AMP/S & IMP & MER & PITA & CFP & AK & GM & CAZ & $\mathrm{CO}$ \\
\hline$A b^{a} . N$ & 8 & 1 & 5 & 1 & 2 & 1 & 0 & 15 & 5 & 0 & 33 \\
\hline (\%) & 24.24 & 3.03 & 15.15 & 3.03 & 6.06 & 3.03 & 0 & 45.45 & 15.15 & 0 & 100 \\
\hline
\end{tabular}

Culture yielded in the Non- $A b$-PJI group were mainly S. aureus, Enterobacter aerogenes, and Pseudomonas aeruginosa. The MRSA rate was $7.0 \%$ (additional file 2).

\section{Outcomes and potential risk factors for Acinetobacter baumannii-associated PJI}

On the univariate analysis, RFs associated with patient characteristics, surgery, and the postoperative period were investigated for possible associations with $A b$-PJI, as shown in Table 2. Compared with PJI caused by other microorganisms, $A b$-PJI was significantly associated with the previous use of antibiotics during the last three months $(51.5 \%$ vs $30.8 \% ; p=0.045)$, previous orthopaedic infections $(36.4 \%$ vs $12.3 \%$; $p=0.005)$, revision arthroplasty $(60.6 \%$ vs $33.8 \% ; p=0.011)$, and post-traumatic/non-elective arthroplasty $(54.4 \%$ vs $32.3 \% ; \mathrm{p}=0.034)$. $A$-PJI patients received more blood transfusions than patients with PJI due to other microorganisms (36.4\% vs $10.8 \% ; p=0.002)$. Early infections occurred in $48.5 \%(16 / 33)$ of patients with $A b$-PJI, and $81.5 \%(53 / 65)$ of patients with Non- $A b$-PJI $(\mathrm{p}=0.001)$. Following an infectious diagnosis, DAIR was the surgical strategy chosen for $57.6 \%$ of the $A b$-PJI cases ( $p=0.047)$, (Table 2$)$. The factors that remained independently associated with $A b$-PJI upon multiple logistic regression were revision arthroplasty $(\mathrm{OR}=3.01 ; 95 \% \mathrm{Cl}=1.15-7.90, \mathrm{p}=0.025)$ and non-elective arthroplasty $(\mathrm{OR}=2.65 ; 95 \% \mathrm{Cl}=1.01-7.01, \mathrm{p}=$ 0.049). In addition, $A b$-PJI was more likely to be classified as a late chronic infection (OR $=5.81 ; 95 \% \mathrm{Cl}=$ $2.1-16.07, \mathrm{p}=0.001$ than Non- $A b-\mathrm{PJI}$. (Table 3) 
Table 2

Univariate analysis of risk factors associated with Ab-PJI ${ }^{a}$.

\section{Variables}

Ab-PJ|a

No. (\%)

$\mathrm{N}=33$

\section{Demographic data}

Age (median [variation]) (years)

Females

\section{Patient-related variables}

Alcoholism

Smoking

Comorbidities (yes)

SAH

$\mathrm{DM}^{\mathrm{d}}$

Malnutrition

Anemia

Cancer

Lung disease

Metabolic syndrome

Cardiovascular disease

Other comorbidities ${ }^{\mathrm{e}}$

ASA score $\mathrm{f}^{\mathrm{f} \text { * }}$

ASA 1

ASA 2

ASA 3
$71.0(48-88)$

$16(48.5)$

7 (21.2)

$4(12.1)$

25 (75.8)

20 (60.6)

$8(24.2)$

4 (12.1)

$2(6.1)$

$0(0.0)$

$0(0.0)$

$5(15.2)$

$2(6.1)$

$3(9.1)$

$2(6.1)$

22 (66.7)

8 (24.2)
NON-Ab-PJ' ${ }^{b}$

No. (\%)

$N=65$

P-value ${ }^{\#}$ 


\begin{tabular}{|c|c|c|c|}
\hline \multirow[t]{3}{*}{ Variables } & Ab-PJIa & NON-Ab-PJlb & \multirow[t]{3}{*}{ P-value } \\
\hline & No. (\%) & \multirow[t]{2}{*}{ No. (\%) } & \\
\hline & $N=33$ & & \\
\hline ASA 4 & $1(3.0)$ & \multicolumn{2}{|l|}{$0(0.0)$} \\
\hline \multicolumn{4}{|l|}{ Use of antimicrobials ${ }^{*}$} \\
\hline Previous use & $17(51.5)$ & $20(30.8)$ & $0.045^{\star}$ \\
\hline Single antimicrobial & $6(18.2)$ & $5(7.7)$ & \multirow[t]{2}{*}{$0.104^{\star \star}$} \\
\hline Antimicrobial combination & $11(33.3)$ & $15(23.1)$ & \\
\hline Previous PJI & $12(36.4)$ & 8 (12.3) & $0.005^{\star}$ \\
\hline \multicolumn{4}{|l|}{ Variables related to the surgical procedure ${ }^{*}$} \\
\hline \multicolumn{4}{|l|}{ Arthroplasty } \\
\hline Total & $24(72.7)$ & $51(78.5)$ & $0.527 *$ \\
\hline Revision & $20(60.6)$ & $22(33.8)$ & $0.011^{*}$ \\
\hline Non-elective & $18(54.4)$ & $21(32.3)$ & $0.034^{*}$ \\
\hline Duration of the procedure $>2.5$ hours & $3(9.1)$ & $12(18.5)$ & $0.223^{*}$ \\
\hline Blood transfusion & $12(36.4)$ & $7(10.8)$ & $0.002^{\star}$ \\
\hline \multicolumn{4}{|c|}{ Variables related to the postoperative period ${ }^{*}$} \\
\hline Infection-related sepsis & $2(6.1)$ & $0(0)$ & $0.111 * \star$ \\
\hline Concomitant non-orthopedic infection & $18.2(6)$ & $10.8(7)$ & $0.352^{\star}$ \\
\hline Surgical wound complications & $42.4(14)$ & $26.6(16)$ & $0.071^{*}$ \\
\hline Early infection & $48.5(16)$ & $81.5(53)$ & $0.001 *$ \\
\hline DAIRg & $57.6(19)$ & $76.9(50)$ & $0.047^{\star}$ \\
\hline \multicolumn{4}{|c|}{ 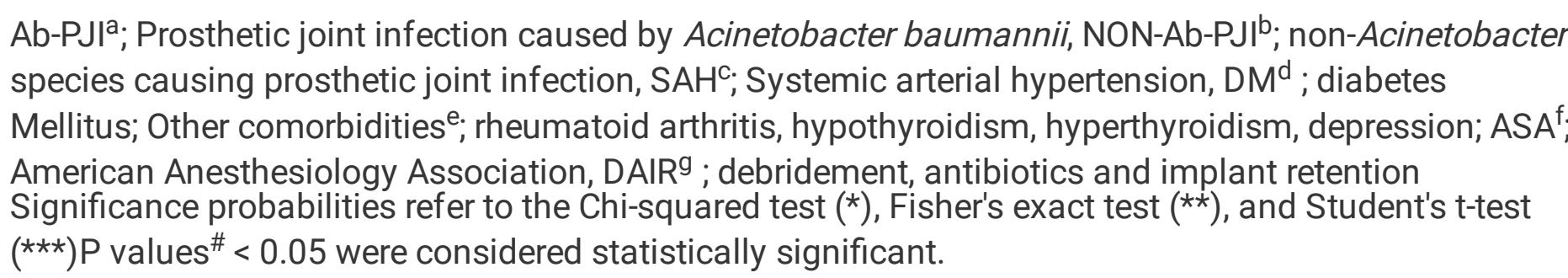 } \\
\hline
\end{tabular}


Table 3

Predisposing factors independently associated to Acinetobacter baumannii PJI -multivariate analysis

\begin{tabular}{|c|c|c|c|c|}
\hline \multirow[t]{3}{*}{ Variables } & A. baumannip & Other bacteria & Odds Ratio & P-value ${ }^{a}$ \\
\hline & No. (\%) & No. (\%) & (OR) & \\
\hline & $N=33$ & $N=65$ & $95 \% \mathrm{Cl}$ & \\
\hline Revision arthroplasty & $20(60.6)$ & $22(33.8)$ & $3.01(1.15-7.90)$ & 0.025 \\
\hline Non-elective arthroplasty & $18(54.5)$ & $21(32.3)$ & $2.65(1.01-7.01)$ & 0.049 \\
\hline Late infection & $17(51.5)$ & $12(18.5)$ & $5.81(2.1-16.07)$ & 0.001 \\
\hline
\end{tabular}

On the KM survival curve, infection by $A b$ was not identified as an RF for treatment failure $(p=0.557$, Fig. 1), and no increase in the failure rate was observed for the Ab-PJI group that underwent DAIR compared with the non Ab-PJI that underwent DAIR $(p=0.530$, Fig. 2).

\section{Discussion}

To our knowledge, this is the first study attempting to investigate predisposing factors associated $A b$-PJI. Interestingly the well-known predictors of PJI, revision surgeries, non-elective arthroplasties and late infections (PJl diagnosed after 3 months of index surgery) were independently associated with $A$. baumannii infection. This likely reflects the particular epidemiology of a Brazilian orthopaedic referral center, on which, the rates of nosocomial SSI caused by MDR-GNB is high [25, 26]. In addition, high selective pressure imposed by misuse of empirical broad-spectrum antibiotics is likely to have played a major role [27]. Since then, a local antimicrobial stewardship program has been implemented as a tool towards the appropriateness of antibiotics prescription.

In the microbiological sample, a higher frequency (81.8\%) of $A b$ strains causing PJI was XDR, whereas $12.1 \%$ were MDR and only $6.1 \%$ were MS. Susceptibility to carbapenems was worryingly low, with only $3 \%$ of cases sensitive to imipenem and $6 \%$ sensitive to meropenem. The higher prevalence of $A b$-PJI at our institution was not assumed to represent an outbreak, but this is rather an endemic nosocomial pathogen typically identified in the intensive care unit (ICU) environment, which presents an overwhelming ability to colonise the human skin. Furthermore, before 2018 the immediate postoperative care for patients who undergo arthroplasty in our hospital was usually performed at ICU, which is likely to have increased the rate of skin colonisation by $A b$ strains.

Orthopaedic implant-associated infections have traditionally been considered a difficult-to-treat disease due to the formation of bacterial biofilms on the implant surface, and the low levels of antibiotic penetration into bone tissue and biofilms. $[28,29]$ In addition, the higher levels of bacterial resistance 
commonly expressed by $A b$, makes treatment even more challenging due to the scarcity of available drugs and to antibiotic-related toxicity.[30] Although $A b$ is ubiquitous in nature and colonises the skin of healthy individuals, most human infections are healthcare-associated. A systematic review by Falagas et al.,[31] which included 55 articles describing $A b$ infections, associated $A b$ infections with prolonged hospital stays, intensive care unit treatments, and the use of invasive devices.

Despite the poor availability of studies specifically describing $A b$-PJI, the number of osteomyelitis and fracture-related infections caused by $A b$ seems to be on the rise worldwide, especially those associated with high-kinetic energy trauma and open fractures.[32] Many studies have described a strong association between complex traumatic gunshot wounds resulting in fracture-related infections or osteomyelitis with $A b$ in various conflict-affected regions, such as Iraq, Afghanistan, and Yemen.[32-35] However, whether $A b$ is acquired during the injury itself from primary contamination, or is hospital-acquired during the trauma care and subsequent surgical procedures remains unclear. In addition to reports from Middle Eastern countries, a study by Vanegas et al [36] from Colombia addressed osteomyelitis, skin and soft tissue infections and reported an increased number of infections caused by $A b$, and strong association with recent hospitalisation or surgery, and previous use of antimicrobials in the past six months. Despite the increased risk of PJI following revision surgeries [37-39], the association between revision arthroplasty and $A b$-PJI has not yet been reported in the literature. However, we are aware that implant contamination during surgery is a primary source of infection, and patients previously colonised with $A b$ may be at increased risk for PJI.

In our study, a 2.6-fold increase in the risk of $A b$-PJI was identified among patients undergoing emergency arthroplasties, which suggests that similar to fracture-related infections, post-traumatic arthroplasties may be a predisposing factor to $A b$-PJI. The reasons underlying the association between trauma and $A b$ infection were not elucidated in this study and require further investigation. In addition, we were unable to identify any independent associations between $A b$-PJI with closed proximal femoral fractures in the elderly population, the recent hospitalisation history, or recent use of antibiotics. However, non-elective arthroplasties may require longer preoperative hospital stays due to the mandatory propaedeutic for assessing preoperative risks and the need to compensate for clinical comorbidities prior to performing the surgical procedure, which might increase the risk of colonisation by $A b$ [40]. However, the length of preoperative hospital stays, which could validate this hypothesis, was not a variable that was assessed in the present study.

In our study late PJI was independently associated with $A b$ infection, and a possible explanation may rely upon the lower level of virulence expression when bacteria expresses multiple antibiotic-resistant mechanisms. Several mechanisms associated with antimicrobial resistance, including pump efflux and biofilm organisation abilities, also decrease the bacterial replicative capacity [41]. This stationary phase, associated with biofilm formation and maturation, is likely to cause $A b$ infections to develop more slowly, increasing the likelihood of being diagnosed as late PJI.[42, 43] Neither $A b$ infections nor the surgical strategy used after the infectious diagnosis was independently associated with final outcomes or risks of treatment failure. Few studies have assessed the prognostic factors associated with $A b$-PJI development, 
although some reported high rates of therapeutic failure in $A b$-PJI, such as Vasso et al., [19] which described a $33.3 \%$ failure rate for $A b$-PJI. However, that study was underpowered, assessing only nine patients in a group containing a mix of infections associated with both $A b$ and $P$. aeruginosa. Another study that assessed the outcomes of PJI caused by BGN-MDR reported that infections caused by MDR/XDR BGN were associated with high-therapeutic failure rates when DAIR (52.2\%) was performed compared with non-DAIR strategies (23.4\%) [44]. However, only 3 patients had $A b$-PJI in this study, which is not a representative sample.

The present study has potential limitations. First, it was performed as a retrospective study, conducted at a single centre, located in a major city in a developing country, which offers specialised orthopaedic care for the regional population. Consequently, the results obtained at our hospital may not apply to other hospitals. In addition, the identification and sensitivity tests were performed using non-automated methods, and no molecular or genotypic analyses were performed to identify clonal variants or similar patterns of resistance mechanisms. Furthermore, no pairing was performed between the $A b$-PJI and Non$A b$-PJI groups to control for preoperative hospitalisation times or the preoperative colonisation by $A b$, which could support the hypothesis that PJI contamination occurred intraoperatively. However, this study explored the previously unexamined issue of PJI-predisposing factors and relied on the largest number of $A b$ infection cases described to date, with a high frequency of MDR/ XDR strains.

\section{Conclusions}

The findings suggest that non-elective and revision arthroplasties that were primarily performed due to trauma, and PJI diagnosed after three months of index surgery were independently associated with $A b$-PJI. IN addition, PJI caused by $A b$ was not associated with treatment failure, and no difference between the DAIR in A $b$-PJI versus NON-A $b$ PJI was identified for disease-free survival rate. The present study added relevant data to the rising field of MDR-GNB PJI cases, but multicentre cohort studies with larger sample size is still needed.

\section{List Of Abbreviations}

- Ab-Acinetobacter baumannii

- ASA - American Society of Anesthesiologists

- BHI - brain-heart infusion

- Cl.- confidence interval

- DAIR - (debridement, antibiotics, and implant retention)

- EUCAST - European Committee on Antimicrobial Susceptibility Testing

- GNB-gram-negative bacilli

- GPC- Gram-positive cocci

- ICU- intensive care unit

- KM-Kaplan-Meier 
- OR- odds ratio

- OXA- oxacillin-hydrolysing

- MRSA- methicillin-resistant Staphylococcus aureus

- MIC- minimum inhibitory concentrations

- MDR- multidrug-resistant

- MSIS- Musculoskeletal Infection Society

- PJI-Prosthetic joint infection

- RF- risk factors

- SD-standard deviation

- SSI- surgical site infections

- WHO- World Health Organization

- XDR - extensively drug-resistant

\section{Declarations}

\section{Ethics approval and consent to participate}

The study was conducted according to the guidelines of the Declaration of Helsinki and approved by Ethics Committee) of Fundação Hospitalar São Francisco de Assis (no. 2,610,914 on April 20, 2018)

\section{Informed Consent Statement}

Patient consent was waived due the research involves no more than minimal risk to the subject because is a retrospect observational study without any intervention. Informed Consent Statement was waived by ethics committee of Fundação Hospitalar São Francisco de Assis. Rua Itamaracá, 535, coordprojetos@saofrancisco.org.br

\section{Consent for publication}

Not applicable

\section{Availability of data and materials}

The datasets generated and/or analysed during the current study are available in the http://www.ensaiosclinicos.gov.br/rg/RBR-6ft5yb/

Register Number: RBR-6ft5yb

\section{Competing interests}

The authors declare no conflict of interest 


\section{Funding}

This research received no external funding

\section{Authors' contributions}

Conceptualization, RBS. and MJCS.; methodology, RBS. and MJCS; validation, MJCS., formal analysis, MJCS.; investigation RBS and ROA.; data curation, RBS.; writing-original draft preparation, RBS and MJCS.; writing-review and editing, MJCS and ROA; visualization, MJCS.; supervision, MJCS.; project administration, RBS. All authors have read and agreed to the published version of the manuscript

\section{Acknowledgements}

We would like to thank the" Fundação Hospitalar São Francisco de Assis" for the support to conduct this study.

\section{References}

1. Kozak LJ, DeFrances CJ, Hall MJ. National hospital discharge survey: 2004 annual summary with detailed diagnosis and procedure data. Vital Health Stat 13. 2006 Oct;(162):1-209. PMID: 17091747

2. Corvec S, Portillo ME, Pasticci BM, Borens O, Trampuz A. Epidemiology and new developments in the diagnosis of prosthetic joint infection. Int J Artif Organs. 2012 Oct;35(10):923-34. doi: 10.5301/ijao.5000168. PMID: 23138706.

3. Ong KL, Kurtz SM, Lau E, Bozic KJ, Berry DJ, Parvizi J. Prosthetic joint infection risk after total hip arthroplasty in the Medicare population. J Arthroplasty. 2009 Sep;24(6 Suppl):105-9. doi: 10.1016/j.arth.2009.04.027. Epub 2009 Jun 2. PMID: 19493644.

4. Martínez-Pastor JC, Muñoz-Mahamud E, Vilchez F, García-Ramiro S, Bori G, Sierra J, Martínez JA, Font $\mathrm{L}$, Mensa J, Soriano A. Outcome of acute prosthetic joint infections due to gram-negative bacilli treated with open debridement and retention of the prosthesis. Antimicrob Agents Chemother. 2009 Nov;53(11):4772-7. doi: 10.1128/AAC.00188-09. Epub 2009 Aug 17. PMID: 19687237; PMCID: PMC2772308.

5. Hsieh PH, Lee MS, Hsu KY, Chang YH, Shih HN, Ueng SW. Gram-negative prosthetic joint infections: risk factors and outcome of treatment. Clin Infect Dis. 2009 Oct 1;49(7):1036-43. doi: 10.1086/605593. PMID: 19691430.

6. Zimmerli W, Trampuz A, Ochsner PE. Prosthetic-joint infections. N Engl J Med. 2004 Oct 14;351(16):1645-54. doi: 10.1056/NEJMra040181. PMID: 15483283.

7. Pradella JGDP, Bovo M, Salles MJC, Klautau GB, Camargo OAP, Cury RPL. Infected primary knee arthroplasty: Risk factors for surgical treatment failure. Rev Bras Ortop. 2013 Oct 18;48(5):432-437. doi: 10.1016/j.rboe.2012.10.006. PMID: 31304148; PMCID: PMC6565953.

8. Nagaya LH, Salles MJC, Takikawa LSC, Fregoneze M, Doneux P, Silva LAD, Sella GDV, Miyazaki AN, Checchia SL. Infections after shoulder arthroplasty are correlated with higher anesthetic risk score: a 
case-control study in Brazil. Braz J Infect Dis. 2017 Nov-Dec;21(6):613-619. doi: 10.1016/j.bjid.2017.06.003. Epub 2017 Jul 10. PMID: 28704642.

9. Peleg AY, Seifert H, Paterson DL. Acinetobacter baumannii: emergence of a successful pathogen. Clin Microbiol Rev. 2008 Jul;21(3):538-82. doi: 10.1128/CMR.00058-07. PMID: 18625687; PMCID: PMC2493088.

10. Longo F, Vuotto C, Donelli G. Biofilm formation in Acinetobacter baumannii. New Microbiol. 2014 Apr;37(2):119-27. Epub 2014 Apr 1. PMID: 24858639.

11. Boucher HW, Talbot GH, Bradley JS, Edwards JE, Gilbert D, Rice LB, Scheld M, Spellberg B, Bartlett J. Bad bugs, no drugs: no ESKAPE! An update from the Infectious Diseases Society of America. Clin Infect Dis. 2009 Jan 1;48(1):1-12. doi: 10.1086/595011. PMID: 19035777

12. CDC Antibiotic resistance Threats in the United States 2019. Atlanta, GA: U.S. Department of Health and Human Services, CDC;2019.

13. Labarca JA, Salles MJ, Seas C, Guzmán-Blanco M. Carbapenem resistance in Pseudomonas aeruginosa and Acinetobacter baumannii in the nosocomial setting in Latin America. Crit Rev Microbiol. 2016;42(2):276-92. doi: 10.3109/1040841X.2014.940494. Epub 2014 Aug 27. PMID: 25159043.

14. Escandón-Vargas K, Reyes S, Gutiérrez S, Villegas MV. The epidemiology of carbapenemases in Latin America and the Caribbean. Expert Rev Anti Infect Ther. 2017 Mar;15(3):277-297. doi: 10.1080/14787210.2017.1268918. Epub 2016 Dec 20. PMID: 27915487.

15. Hischebeth GT, Wimmer MD, Molitor E, Seifert H, Gravius S, Bekeredjian-Ding I. Multidrug resistant Acinetobacter baumannii reaches a new frontier: prosthetic hip joint infection. Infection. 2015 Feb;43(1):95-7. doi: 10.1007/s15010-014-0661-x. Epub 2014 Jul 19. PMID: 25037735.

16. Krzyściak P, Chmielarczyk A, Pobiega M, Romaniszyn D, Wójkowska-Mach J. Acinetobacter baumannii isolated from hospital-acquired infection: biofilm production and drug susceptibility. APMIS. 2017 Nov;125(11):1017-1026. doi: 10.1111/apm.12739. Epub 2017 Sep 15. PMID: 28913903.

17. Runci F, Bonchi C, Frangipani E, Visaggio D, Visca P. Acinetobacter baumannii Biofilm Formation in Human Serum and Disruption by Gallium. Antimicrob Agents Chemother. 2016 Dec 27;61(1): e0156316. doi: 10.1128/AAC.01563-16. PMID: 27799219; PMCID: PMC5192145.

18. Vila A, Pagella H, Amadio C, Leiva A. Acinetobacter Prosthetic Joint Infection Treated with Debridement and High-Dose Tigecycline. Infect Chemother. 2016 Dec;48(4):324-329. doi: 10.3947/ic.2016.48.4.324. Epub 2016 Nov 8. PMID: 27883369; PMCID: PMC5204012.

19. Vasso M, Schiavone Panni A, De Martino I, Gasparini G. Prosthetic knee infection by resistant bacteria: the worst-case scenario. Knee Surg Sports Traumatol Arthrosc. 2016 Oct;24(10):3140-3146. doi: 10.1007/s00167-016-4010-8. Epub 2016 Feb 1. PMID: 26831859.

20. Parvizi J, Zmistowski B, Berbari EF, Bauer TW, Springer BD, Della Valle CJ, Garvin KL, Mont MA, Wongworawat MD, Zalavras CG. New definition for periprosthetic joint infection: from the Workgroup of the Musculoskeletal Infection Society. Clin Orthop Relat Res. 2011 Nov;469(11):2992-4. doi: 10.1007/s11999-011-2102-9. PMID: 21938532; PMCID: PMC3183178. 
21. Magiorakos AP, Srinivasan A, Carey RB, Carmeli Y, Falagas ME, Giske CG, Harbarth S, Hindler JF, Kahlmeter G, Olsson-Liljequist B, Paterson DL, Rice LB, Stelling J, Struelens MJ, Vatopoulos A, Weber JT, Monnet DL. Multidrug-resistant, extensively drug-resistant and pandrug-resistant bacteria: an international expert proposal for interim standard definitions for acquired resistance. Clin Microbiol Infect. 2012 Mar;18(3):268-81. doi: 10.1111/j.1469-0691.2011.03570. x. Epub 2011 Jul 27. PMID: 21793988.

22. Kandel CE, Jenkinson R, Daneman N, Backstein D, Hansen BE, Muller MP, Katz KC, Widdifield J, Bogoch E, Ward S, Sajja A, Jeldes FG, McGeer A. Predictors of Treatment Failure for Hip and Knee Prosthetic Joint Infections in the Setting of 1- and 2-Stage Exchange Arthroplasty: A Multicenter Retrospective Cohort. Open Forum Infect Dis. 2019 Oct 21;6(11): ofz452. doi: 10.1093/ofid/ofz452. PMID: 31737739; PMCID: PMC6847009.

23. Shohat N, Goswami K, Tan TL, Fillingham Y, Parvizi J. Increased Failure After Irrigation and Debridement for Acute Hematogenous Periprosthetic Joint Infection. J Bone Joint Surg Am. 2019 Apr 17;101(8):696-703. doi: 10.2106/JBJS.18.00381. PMID: 30994587.

24. Eclercq R, Cantón R, Brown DF, Giske CG, Heisig P, MacGowan AP, Mouton JW, Nordmann P, Rodloff AC, Rossolini GM, Soussy CJ, Steinbakk M, Winstanley TG, Kahlmeter G. EUCAST expert rules in antimicrobial susceptibility testing. Clin Microbiol Infect. 2013 Feb;19(2):141-60. doi: 10.1111/j.14690691.2011.03703. x. Epub 2011 Nov 25. PMID: 22117544.

25. Villegas MV, Blanco MG, Sifuentes-Osornio J, Rossi F. Increasing prevalence of extended-spectrumbeta-lactamase among Gram-negative bacilli in Latin America-2008 update from the Study for Monitoring Antimicrobial Resistance Trends (SMART). Braz J Infect Dis. 2011 Jan-Feb;15(1):34-9. PMID: 21412587.

26. Gales AC, Castanheira M, Jones RN, Sader HS. Antimicrobial resistance among Gram-negative bacilli isolated from Latin America: results from SENTRY Antimicrobial Surveillance Program (Latin America, 2008-2010). Diagn Microbiol Infect Dis. 2012 Aug;73(4):354-60. doi:

10.1016/j.diagmicrobio.2012.04.007. Epub 2012 May 31. PMID: 22656912.

27. Davies J, Davies D. Origins and evolution of antibiotic resistance. Microbiol Mol Biol Rev. 2010 Sep;74(3):417-33. doi: 10.1128/MMBR.00016-10. PMID: 20805405; PMCID: PMC2937522.

28. del Pozo JL, Patel R. The challenge of treating biofilm-associated bacterial infections. Clin Pharmacol Ther. 2007 Aug;82(2):204-9. doi: 10.1038/sj.clpt.6100247. Epub 2007 May 30. PMID: 17538551.

29. Saunders RK Jr, Infanti J, Ali H, Shuey T, Potteiger C, McNeilly S, Adams CS. Gram-Negative Bacteria Are Internalized Into Osteocyte-Like Cells. J Orthop Res. 2020 Apr;38(4):861-870. doi:

10.1002/jor.24510. Epub 2019 Nov 17. PMID: 31692074.

30. Siljander MP, Sobh AH, Baker KC, Baker EA, Kaplan LM. Multidrug-Resistant Organisms in the Setting of Periprosthetic Joint Infection-Diagnosis, Prevention, and Treatment. J Arthroplasty. 2018 Jan;33(1):185-194. doi: 10.1016/j.arth.2017.07.045. Epub 2017 Aug 3. PMID: 28869114.

31. Falagas ME, Kopterides P. Risk factors for the isolation of multi-drug-resistant Acinetobacter baumannii and Pseudomonas aeruginosa: a systematic review of the literature. J Hosp Infect. 2006 Sep;64(1):7-15. doi: 10.1016/j.jhin.2006.04.015. Epub 2006 Jul 5. PMID: 16822583. 
32. Schafer JJ, Mangino JE. Multidrug-resistant Acinetobacter baumannii osteomyelitis from Iraq. Emerg Infect Dis. 2008 Mar;14(3):512-4. doi: 10.3201/eid1403.070128. PMID: 18325278; PMCID: PMC2570836.

33. Murphy RA, Ronat JB, Fakhri RM, Herard P, Blackwell N, Abgrall S, Anderson DJ. Multidrug-resistant chronic osteomyelitis complicating war injury in Iraqi civilians. J Trauma. 2011 Jul;71(1):252-4. doi: 10.1097/TA.0b013e31821b8622. PMID: 21818032.

34. Yun HC, Branstetter JG, Murray CK. Osteomyelitis in military personnel wounded in Iraq and Afghanistan. J Trauma. 2008 Feb;64(2 Suppl):S163-8; discussion S168. doi: 10.1097/TA.0b013e318160868c. PMID: 18376160.

35. Lohr B, Pfeifer Y, Heudorf U, Rangger C, Norris DE, Hunfeld KP. High Prevalence of Multidrug-Resistant Bacteria in Libyan War Casualties Admitted to a Tertiary Care Hospital, Germany. Microb Drug Resist. 2018 Jun;24(5):578-584. doi: 10.1089/mdr.2017.0141. Epub 2017 Oct 17. PMID: 29039717.

36. Vanegas JM, Higuita LF, Vargas CA, Cienfuegos AV, Rodríguez ÉA, Roncancio GE, Jiménez JN. Acinetobacter baumannii resistente a carbapenémicos causante de osteomielitis e infecciones de la piel y los tejidos blandos en hospitales de Medellín, Colombia [Carbapenem-resistant Acinetobacter baumannii causing osteomyelitis and infections of skin and soft tissues in hospitals of Medellín, Colombia]. 2015 Oct-Dec;35(4):522-30. Spanish. doi: 10.7705/biomedica.v35i4.2572. PMID: 26844441.

37. Jacobs AME, Bénard M, Meis JF, van Hellemondt G, Goosen JHM. The unsuspected prosthetic joint infection: incidence and consequences of positive intra-operative cultures in presumed aseptic knee and hip revisions. Bone Joint J. 2017 Nov;99-B(11):1482-1489. doi: 10.1302/0301-620X.99B11.BJJ2016-0655.R2. PMID: 29092987.

38. Hoell S, Moeller A, Gosheger G, Hardes J, Dieckmann R, Schulz D. Two-stage revision arthroplasty for periprosthetic joint infections: What is the value of cultures and white cell count in synovial fluid and CRP in serum before second stage reimplantation? Arch Orthop Trauma Surg. 2016 Apr;136(4):44752. doi: 10.1007/s00402-015-2404-6. Epub 2016 Jan 12. PMID: 26757939.

39. Kunutsor SK, Whitehouse MR, Blom AW, Beswick AD; INFORM Team. Patient-Related Risk Factors for Periprosthetic Joint Infection after Total Joint Arthroplasty: A Systematic Review and Meta-Analysis. PLoS One. 2016 Mar 3;11(3):e0150866. doi: 10.1371/journal.pone.0150866. PMID: 26938768; PMCID: PMC4777569.

40. Thorne A, Luo T, Durairajan NK, Kaye KS, Foxman B. Risk factors for endemic Acinetobacter Baumannii colonization: A case-case study. Am J Infect Control. 2019 Nov;47(11):1294-1297. doi: 10.1016/j.ajic.2019.04.179. Epub 2019 Jun 26. PMID: 31253551.).

41. Blair JM, Webber MA, Baylay AJ, Ogbolu DO, Piddock LJ. Molecular mechanisms of antibiotic resistance. Nat Rev Microbiol. 2015 Jan;13(1):42-51. doi: 10.1038/nrmicro3380. Epub 2014 Dec 1. PMID: 25435309).

42. Salles MJ, Zurita J, Mejía C, Villegas MV; Latin America Working Group on Bacterial Resistance. Resistant gram-negative infections in the outpatient setting in Latin America. Epidemiol Infect. 2013 
Dec;141(12):2459-72. doi: 10.1017/S095026881300191X. Epub 2013 Aug 7. PMID: 23924513; PMCID: PMC3821403.

43. Dai J, Jiang C, Chen H, Chai Y. Assessment of the Risk Factors of Multidrug-Resistant Organism Infection in Adults With Type 1 or Type 2 Diabetes and Diabetic Foot Ulcer. Can J Diabetes. 2020 Jun;44(4):342-349. doi: 10.1016/j.jcjd.2019.10.009. Epub 2019 Nov 9. PMID: 32005564.

44. Papadopoulos A, Ribera A, Mavrogenis AF, Rodriguez-Pardo D, Bonnet E, JoséSalles M, Dolores Del Toro M, Nguyen S, Blanco-García A, Skaliczki G, Soriano A, Benito N, Petersdorf S, Pasticci MB, Tattevin P, Tufan ZK, Chan M, O'Connell N, Pantazis N, Kyprianou A, Pigrau C, Megaloikonomos PD, Senneville E, Ariza J, Papagelopoulos PJ, Giannitsioti E; ESCMID Study Group for Implant-Associated Infections (ESGIAI). Corrigendum to "Multidrug-resistant and extensively drug-resistant Gram-negative prosthetic joint infections: Role of surgery and impact of colistin administration" [International Journal of Antimicrobial Agents 53(3) (2019) 294-301]. Int J Antimicrob Agents. 2019 Apr;53(4):538-539. doi: 10.1016/j.ijantimicag.2019.03.005. Epub 2019 Mar 23. Erratum for: Int J Antimicrob Agents. 2019 Mar,53(3):294-301. PMID: 30910479.

\section{Figures}

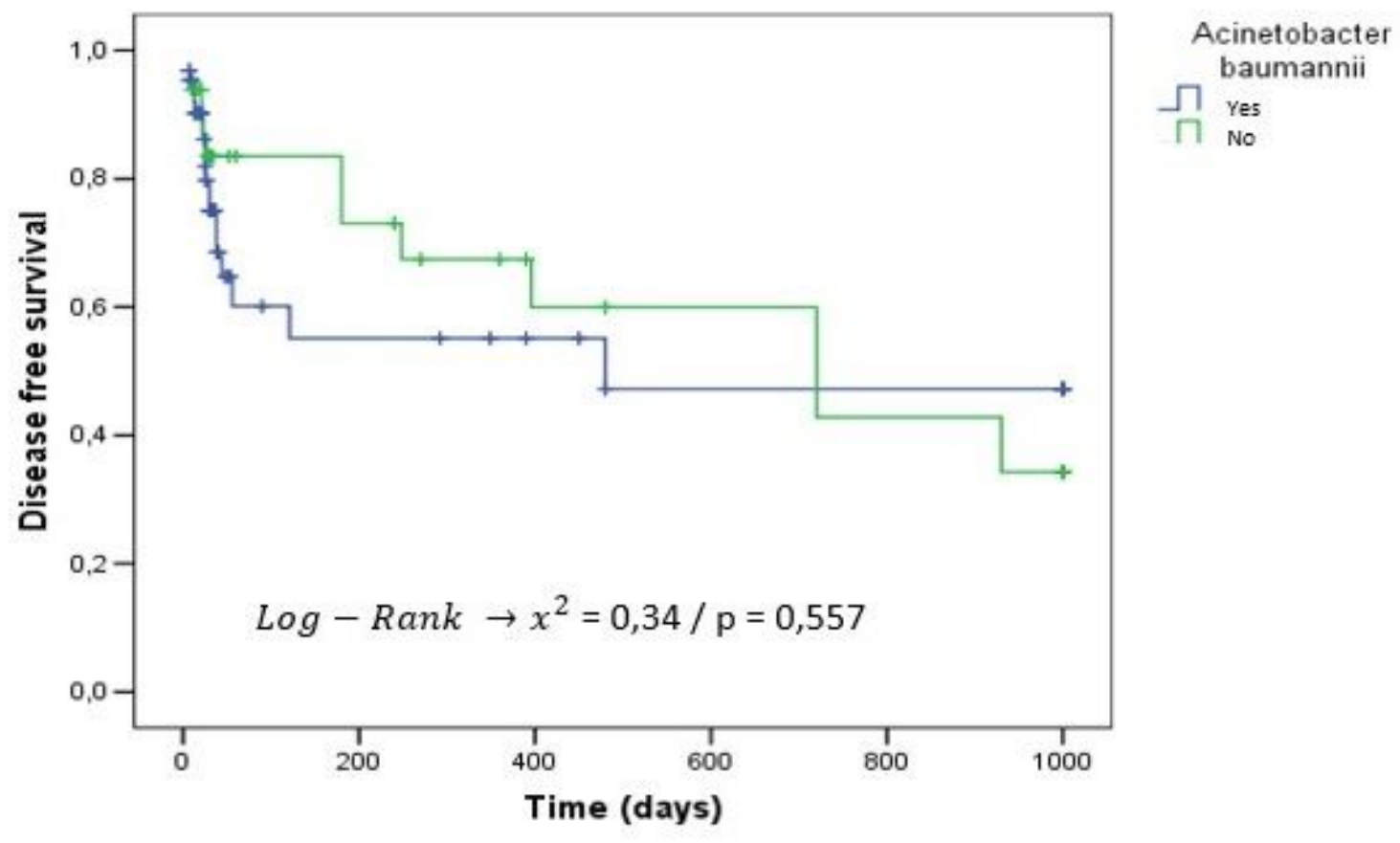

\section{Figure 1}

Kaplan-Meier survival curve for death/recurrence considering PJI caused by Acinetobacter baumannii 


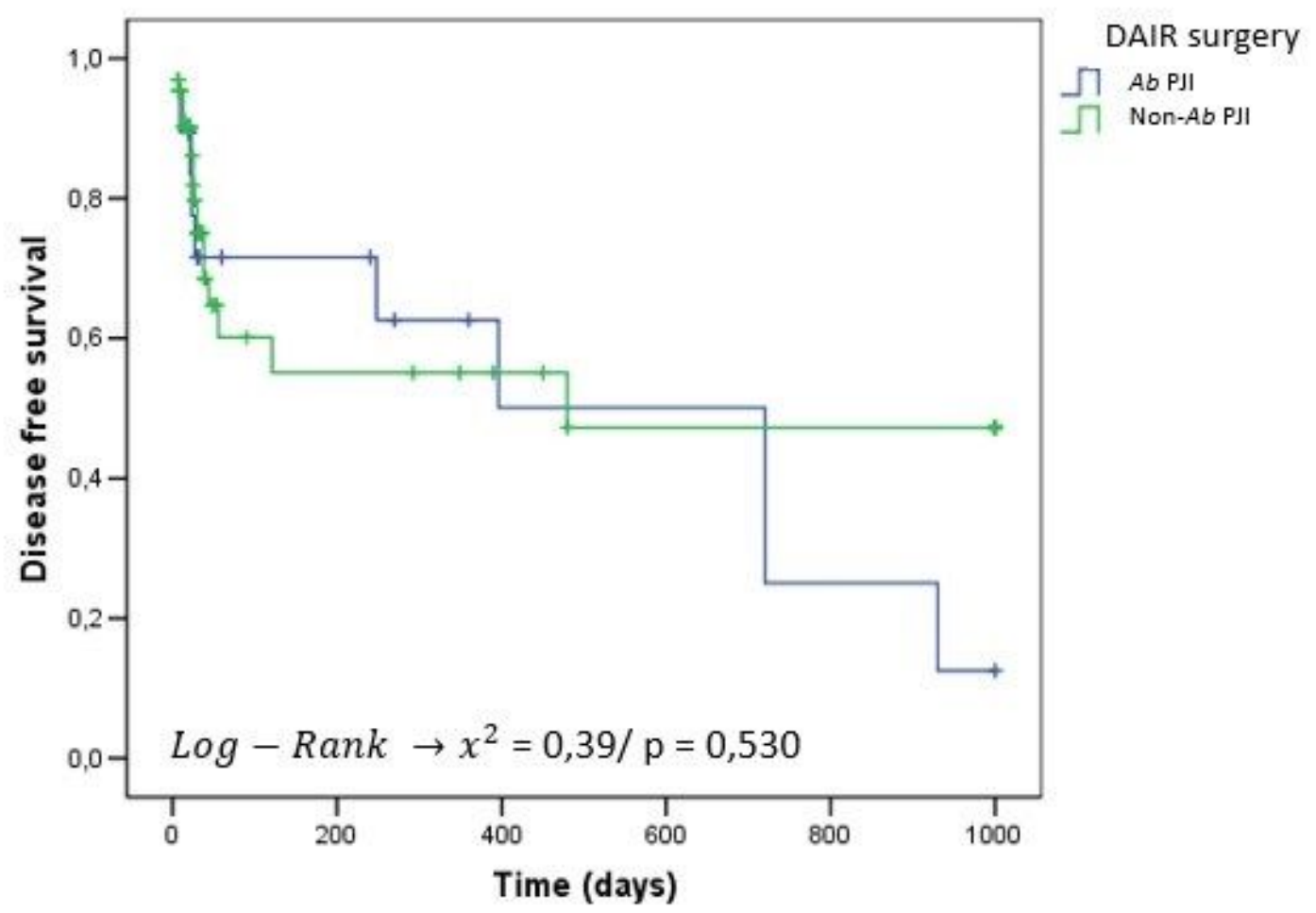

Figure 2

Kaplan-Meier survival curve for death/recurrence considering PJI by A. baumannii PJI DAIR versus Non-AbPJI DAIR

\section{Supplementary Files}

This is a list of supplementary files associated with this preprint. Click to download.

- 20.04Additionalfile.docx 